\title{
Staged unilateral lung volume reduction surgery: from mini-invasive to minimalist treatment strategies
}

\author{
Eugenio Pompeo ${ }^{1}$, Paola Rogliani ${ }^{2}$, Benedetto Cristino ${ }^{1}$, Eleonora Fabbi ${ }^{3}$, Mario Dauri ${ }^{3}$, Gianluigi Sergiacomi ${ }^{4}$ \\ ${ }^{1}$ Department of Thoracic Surgery, ${ }^{2}$ Department of Respiratory Medicine, ${ }^{3}$ Department of Anesthesia and Intensive Care, ${ }^{4}$ Department of Radiology, \\ Policlinico Tor Vergata University, Rome, Italy \\ Contributions: (I) Conception and design: E Pompeo; (II) Administrative support: All authors; (III) Provision of study materials or patients: E Pompeo, \\ M Dauri, P Rogliani; (IV) Collection and assembly of data: B Cristino; (V) Data analysis and interpretation: E Pompeo; (VI) Manuscript writing: All \\ authors; (VII) Final approval of manuscript: All authors. \\ Correspondence to: Eugenio Pompeo, MD, FETCS. Department of Thoracic Surgery, Policlinico Tor Vergata University, V.le Oxford, 81, Rome \\ 00133, Italy. Email: pompeo@med.uniroma2.it.
}

\begin{abstract}
Lung volume reduction surgery (LVRS) entailing unilateral or bilateral non-anatomical resection of severely damaged emphysematous tissue carried out by thoracoscopic or open surgical approaches, under general anesthesia with single-lung ventilation, has resulted in significant and longlasting clinical and functional benefit. Unfortunately, the morbidity rates reported by simultaneous bilateral resectional LVRS has led to raise criticism regarding its cost-effectiveness and has stimulated in recent years the development of less invasive bronchoscopic and surgical non-resectional methods of treatment that are preferentially performed in a staged unilateral fashion. We had previously proposed an innovative LVRS modality, which did not entail any resection of lung tissue and was electively carried out according to a staged unilateral strategy by a multiport thoracoscopic access, through thoracic epidural anesthesia in conscious, spontaneously ventilating patients (awake LVRS). The awake LVRS resulted in significant clinical benefit paralleling that achieved by the resectional method with lower morbidity rates and shorter hospital stay. Moreover, the awake LVRS proved also suitable to be employed in stringently selected patients to perform redo procedures following previous successful bilateral LVRS. More recently, in order to minimize the global surgery- and anesthesia-related traumas, we have modified our original non-resectional method by adopting a single thoracoscopic access as well as an anesthesia protocol entailing use of a simple intercostal block with target control sedation, to realize an ultra-minimally invasive or minimalist LVRS. Hence, a deeper investigation of the pros and cons of staged unilateral LVRS strategies as well as of the novel surgical non-resectional and redo LVRS is warranted in order to verify, the optimal strategies of treatment, which will prove to reduce the typical LVRS-related morbidity while assuring the most durable benefit in patients with advanced emphysema.
\end{abstract}

Keywords: Lung volume reduction surgery (LVRS); emphysema; video-assisted thoracic surgery (VATS); nonintubated anesthesia; thoracoscopy; chronic obstructive pulmonary disease (COPD)

Submitted Feb 17, 2018. Accepted for publication May 21, 2018.

doi: $10.21037 /$ jtd.2018.05.171

View this article at: http://dx.doi.org/10.21037/jtd.2018.05.171

\section{Introduction}

Pulmonary emphysema is a highly disabling chronic condition characterized by a progressive destruction of lung tissue leading to the development of enlarged airspaces denominated bullae, which result in reduced area for gas exchange and impaired elastic recoil of the lung inducing premature collapse of the small airways during expiration, gas trapping, and pulmonary hyperinflation. The sum of detrimental effects related to the progression of emphysema 
eventually compromises pulmonary function, quality of life and overall survival $(1,2)$. In advanced stages of disease, medical therapy is poorly effective and interventional treatment including lung volume reduction surgery (LVRS) can be advocated. Indeed, LVRS entailing simultaneous bilateral non-anatomical resection of severely destroyed lung regions, performed through open or thoracoscopic (3) approaches, has offered survival benefit and significant improvements in respiratory function, exercise capacity and quality of life measures. The clinical-functional benefit achieved by LVRS has resulted of greater magnitude in candidates with heterogeneous distribution of emphysema and upper-lobe predominance of the disease, associated with low exercise capacity (4-6). Unfortunately, in a large multi-institutional controlled study, one-stage bilateral resectional LVRS has resulted in mortality and morbidity rates of $5 \%$ and $59 \%$, respectively, leading to reconsider its cost-effectiveness (6).

In an attempt of reducing some of the potential adverse effects of simultaneous bilateral resectional LVRS, a role for staged unilateral strategy of treatment (7-13) associated with adoption of novel non-resectional surgical $(14,15)$ and bronchoscopic (16-20) less invasive treatment methods has been hypothesized (21).

Our surgical strategy has evolved over time. We had previously proposed an innovative LVRS modality, which did not entail any resection of lung tissue and was electively carried out according to a staged unilateral strategy by a multiport thoracoscopic access, through thoracic epidural anesthesia in conscious, spontaneously ventilating patients (awake LVRS). Our current surgical strategy is based on a modified non-resectional LVRS method that is preferentially carried out in a staged unilateral fashion through a single thoracoscopic access, by a nonintubated anesthesia protocol with maintenance of spontaneous ventilation, in order to realize an intentional ultra-miniinvasive or minimalist surgical treatment of advanced emphysema patients.

In this review we report on technical details and results of staged unilateral LVRS according to our own experience as well as of those drawn from other literature reports.

\section{Selection criteria}

\section{Inclusion criteria}

LVRS can be considered in patients with chronic obstructive pulmonary disease (COPD) of the emphysematous phenotype who are severely symptomatic despite maximized medical treatment and respiratory rehabilitation (22).

Overall, candidates for LVRS should be younger than 80 years and should disclose a moderate to severe obstructive ventilatory defect as shown by an forced expiratory volume in one second $\left(\mathrm{FEV}_{1}\right)<50 \%$ at spirometry associated with significant signs of gas trapping and lung hyperinflation as shown by a plethysmographic residual volume (RV) $>150 \%$ and of flat diaphragms on chest $\mathrm{X}$-rays.

Regarding radiologic morphology of the emphysema, high resolution computed tomography (HRCT) should preferentially disclose recognizable within-lung heterogeneous emphysema pattern characterized by severely destroyed lung regions intermingled with better preserved lung tissue (Figure 1). In this regard, upper lobe-predominant emphysema associated with limited exercise capacity have shown to represent the ideal prerequisites for LVRS in terms of expected magnitude and duration of the clinical benefit (6). Nonetheless, it is recommended that patients should be able to run a six-minute walking test (6MWT) distance $>200 \mathrm{~m}$ to avoid excessive risks of morbidity.

Oxygenation criteria include an arterial oxygen tension $\left(\mathrm{PaO}_{2}\right)>45 \mathrm{mmHg}$ on room air and a carbon dioxide tension $\left(\mathrm{PaCO}_{2}\right)<55-60 \mathrm{mmHg}$ at blood gas analysis.

Stable abstinence from cigarette smoking since at least 3 months is recommended to reduce morbidity risks and confirm the patient's motivation to undergo the surgical treatment.

As far as indications for staged unilateral treatment are regarded, we have found that an asymmetric distribution (distinct between-lungs heterogeneity) of emphysema as indicated by radiological evidence of unilaterally predominant lung destruction and/or hyperinflation, can be associated with postoperative peak benefits, which parallel those achievable by simultaneous bilateral treatment in subjects with symmetrical pattern of disease distribution $(23,24)$.

\section{Exclusion criteria}

HRCT evidence of homogeneous emphysema has been considered a contraindication for LVRS though satisfactory results can be achieved even in this patient sub-cohort (25). Instead, the association of extremely impaired lung function as indicated by $\mathrm{FEV}_{1} \leq 20 \%$ predicted combined with either homogeneous emphysema or diffusion capacity for carbon monoxide (DLCO) $\leq 20 \%$ predicted, have been reported to be associated with a mortality rate as high as $16 \%$ (26) and should thus be considered contraindications for LVRS. 

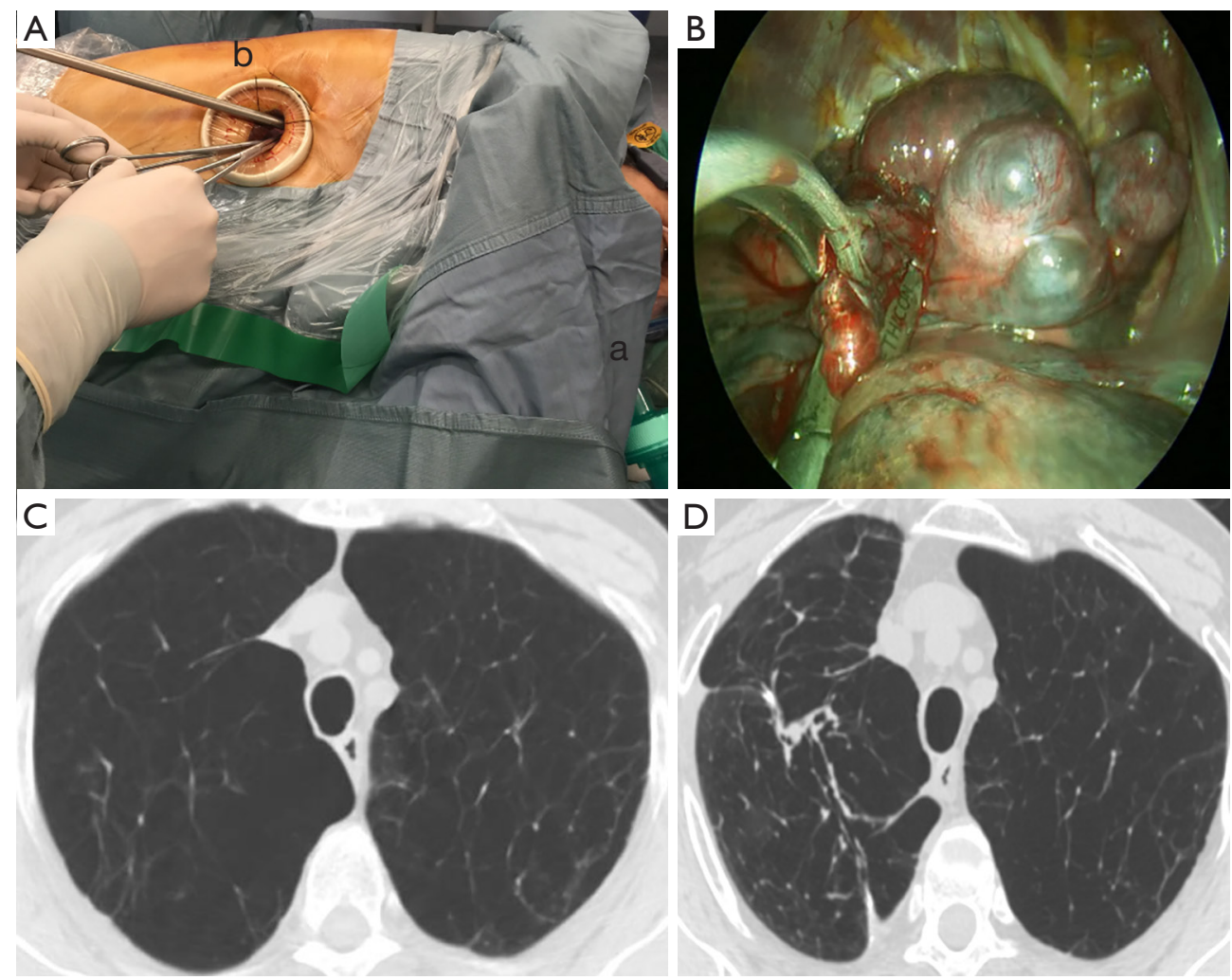

Figure 1 Operative details and radiological outcome of a right-sided minimalist LVRS procedure. (A) Operative view of the minimalist LVRS method showing the single-access approach including adoption of a laryngeal mask (a) and of a silk suture crossing the wound-covering port to fix the camera in upward position and facilitating surgical maneuvering (b); (B) a large plication is being accomplished by peripheral noknife stapling starting in the middle of the lung upper lobe; preoperative (C) versus postoperative (D) computed tomography scan of the chest demonstrate that following minimalist LVRS, a significant reduction of the right lung volume is achieved with any loss of tissue. LVRS, lung volume reduction surgery.

Independent by the severity of emphysema on HRCT, excessive daily sputum production with frequent COPD exacerbations indicating coexistence of severe chronic bronchitis are contraindication for LVRS since this condition can jeopardize the beneficial effects of LVRS on ventilatory mechanics. In this respect, assessment of inspiratory resistance of the airways, has been suggested as a marker of the bronchitis component, with high values deemed to predict a poor response to LVRS (27). Nonetheless, inspiratory resistance and HRCT measures of the airway disease did not predict improvements in either $\mathrm{FEV}_{1}$ or maximal exercise in another report (28).

Recognized severe co-morbid conditions that are likely to predict a limited survival expectation or the impossibility to undergo an aggressive postoperative rehabilitation program are also deemed criteria for exclusion. In addition, history or clinical evidence of ischemic cardiac disease, mandates a thorough investigation including pharmacologic stress test and if necessary, coronary angiography, to exclude the coexistence of coronary artery disease, which can have a high prevalence in patients with advanced emphysema (29).

\section{Staged unilateral resectional LVRS}

So far, no randomized study has compared results of staged unilateral versus one-stage bilateral LVRS (9). In previous retrospective studies, the hospitalization time resulting from the sum of time spent into the hospital for each procedure to complete a staged unilateral treatment with a 3-to 4 months interval, was longer than that required for onestage bilateral LVRS (30).

Nonetheless, the steeper postoperative decay in $\mathrm{FEV}_{1}$ reported with simultaneous bilateral LVRS when compared with that of unilateral procedures (31) and the possibility that unilateral treatment might positively affect the function of both lungs due to the relative mobility of 
the mediastinum (32), have supported a role for staged unilateral strategies of treatment.

Our group, in a comparative analysis of staged versus one-stage bilateral thoracoscopic LVRS (13) in which the completion of the bilateral treatment was performed at the reappearance of severe disability after a mean time of 15.2 months, has shown that although peak improvements were greater in the one-stage group, improvements in $\mathrm{FEV}_{1}$, forced vital capacity (FVC), RV, and 6MWT distance was more stable in the staged group. In accordance with these findings, at 36 months, $\mathrm{RV}$ was found to be significantly lower in the staged group (4.6 versus $5.3 \mathrm{~L}$, $\mathrm{P}=0.01)$.

In another series of 97 patients undergoing intentional unilateral thoracoscopic LVRS (23), we have shown that at 5 years, $70 \%$ of operated patients did not need yet of contralateral treatment suggesting that in many patients, particularly when radiologic evidence of distinct betweenlungs heterogeneity of emphysema is recognized at the HRCT, the completion of a staged bilateral treatment can be delayed of several years or even definitively avoided.

In accordance with our findings, Oey and coworkers (10) analyzed results of a 114 patients cohort undergoing onestage bilateral (26 patients) or unilateral (88 patients) LVRS that included the possibility of a staged unilateral treatment performed on patient request. At a median follow-up of 2.8 years, a staged bilateral operation was performed in 16 patients only with a median time interval between the procedures of 3.9 years. There was no intergroup difference in operative mortality and 5-year survival. However, assessment of quality of life domains according to the 36item short-form quality of life questionnaire (SF-36) were still significantly improved at 4 and 6 years, respectively, in the staged group only. In a more recent update of the same series (33) out of 292 LVRS procedures, 268 were performed thoracoscopically. Bilateral operations were carried out in one-stage in 13 patients and in a staged fashion in 37. Overall median hospital stay was of 13 days and operative mortality was $4.3 \%$. In this study a longer hospitalization was associated with both advanced age and air leak duration, which in turn was related to diffusion capacity and $\mathrm{FEV}_{1}$.

In a small recent series from Zhang and coworkers (34) results of 11 staged bilateral, uniportal thoracoscopic LVRS is reported. Postoperative mean hospital stay was about 16 days whereas morbidity regarded 5 cases of persistent air leakage and 7 cases of pneumonia. With a mean follow-up ranging between 3 up to 12 months, the pulmonary function, $\mathrm{PaO}_{2}, 6 \mathrm{MWT}$ distance and quality of life were improved following either unilateral and bilateral procedures. Moreover, no significant difference between results of unilateral and bilateral procedures was found in terms of improvements in quality of life measures.

\section{Non-resectional LVRS}

Historical background of non-resectional LVRS include the surgical method developed by Crosa-Dorado et al. (35) in 1992 and entailing multiple fold plication of emphysematous lung regions performed through thoracotomy under general anesthesia. This original technique was subsequently slightly modified by Swanson et al. (36) for an easier videoassisted thoracic surgery (VATS) application.

In 2006, our group (14) reported on feasibility and preliminary results of an innovative thoracoscopic nonresectional LVRS method entailing plication of the most severely destroyed target lung areas, carried out through thoracic epidural anesthesia in spontaneously ventilating, conscious patients (denominated awake LVRS). This method, which included interrupted peripheral staple suturing and inlay pleural buttress of the suture line allowed to achieve significant clinical benefits with no mortality, minor morbidity and reduced incidence of prolonged air leaks.

Subsequently, we performed a retrospective comparative analysis of perioperative outcome between 66 patients treated by the awake non-resectional LVRS versus 66 patients undergoing non-awake resectional LVRS (37). Air-leak lasting $>7$ days occurred in a significantly lower proportion of patients in the awake group as compared with results in the control group (18\% vs. $40 \%, \mathrm{P}=0.007)$. As a result, hospital stay also was shorter in the awake group (6.3 days vs. 9.2 days, $\mathrm{P}<0.0001$ ). In another 42 patients' series in which intermediate-term results of the awake LVRS method was assessed (38), there was no 90-day mortality and significant improvements occurred at 24 months in 6MWT, $\mathrm{FEV}_{1}, \mathrm{FVC}, \mathrm{RV}$ as well as in the multidimensional body mass index, airflow obstruction, dyspnea, and exercise capacity index (BODE), which has been found to represent a useful predictor of survival in COPD patients.

In a more recent unicenter trial (15), 63 patients were randomly allocated to receive unilateral thoracoscopic LVRS by either the awake non-resectional (32 patients) or the non-awake resectional (31 patients) method. Comparative outcome assessment between the awake and nonawake groups have shown that $1 \mathrm{~h}$ after surgery, the $\mathrm{PaO}_{2} / \mathrm{FiO}_{2}$ ratio and $\mathrm{PaCO}_{2}$, were significantly better in the 
awake group. Mortality was 0 vs. $3.2 \%$ with no significant intergroup difference whereas morbidity rate was lower in the awake group $(22 \%$ vs. $52 \%, \mathrm{P}<0.01)$. Median hospital stay also was shorter in the awake group ( 6 vs. 7.5 days, $\mathrm{P}<0.04)$ and in a similar manner, more patients in the awake group could be discharged within 6 days (21 versus 10 patients, $\mathrm{P}=0.01$ ). As far as clinical outcome was regarded, $\mathrm{FEV}_{1}$, which was the primary clinical outcome measure, improved significantly between 6 and 24 months in both study groups $(0.28 v s .0 .29 \mathrm{~L})$ with no intergroup difference. Moreover, other measures including 6MWT, FVC, $\mathrm{RV}$ and physical functioning SF-36 domain score, also improved significantly after surgery for up to 24 months. At 36 months, survival and freedom from opposite LVRS treatment did not differ significantly $(\mathrm{P} \geq 0.5)$ between the awake and the control group (81\% versus $87 \%$ and $55 \%$ versus $50 \%$, respectively).

\section{Redo LVRS}

Redo LVRS has been selectively performed in unilateral or staged bilateral fashion in patients who had already undergone previous successful bilateral LVRS but have eventually lost the postoperative clinical improvements and have developed new emphysematous lung areas amenable of a reoperation. In these instances, the postoperative functional deterioration that follows an initial peak improvement, usually progresses along several years. As a result, for quite many of these delicate patients, redo-LVRS remains the only therapeutic choice provided that many of them are older than 65 years and thus cannot be included in a waiting list for lung transplantation.

In 2008, we had reported a series (39) of 17 patients who underwent iterative LVRS procedures entailing completion lobectomy in 7 patients and intubated resectional or awake non-resectional redo LVRS in 5 patients each. In this series, patients' mean age was 66 years and a mean time of 55 months passed from the initial bilateral LVRS and the redo procedure. Overall mortality at 90 -days was $12 \%$, hospital stay averaged 9 days and significant improvements in $\mathrm{FEV}_{1}(\mathrm{P}<0.001)$, FVC $(\mathrm{P}<0.002), \mathrm{RV}(\mathrm{P}<0.001), 6 \mathrm{MWT}$ $(\mathrm{P}<0.001)$, and dyspnea index $(\mathrm{P}<0.001)$ occurred early after surgery and remained relatively stable for up to 12 months.

In another more recent series from Kostron and coworkers (40), 22 patients (9 females) with severe emphysema underwent redo-LVRS following simultaneous bilateral LVRS with a median interval time between the procedures of 60 months. Redo-LVRS was performed unilaterally and by thoracoscopy in 19 patients whereas by thoracotomy in other 3 patients with evidence of severe pleural adhesions. There was no 90-day mortality in this series but one patient died 15 months after surgery. The most frequent complication regarded the development of prolonged air leaks with a median drainage time of 11 days. In 7 of these patients, a further reoperation due to persistent air leak was required. Lung function and Medical Research Council (MRC) score improved significantly for up to 12 months. On average, at 3 months, $\mathrm{FEV}_{1}$ increased by $0.18 \mathrm{~L}$ or $25 \%$, the mean reduction in $\mathrm{RV} /$ total lung capacity was of $12 \%$ whereas the MRC dyspnea index decreased significantly from a score of 3.7 to 2.2 .

\section{The minimalist LVRS strategy}

At our Institution, we have rapidly switched from simultaneous bilateral LVRS to staged unilateral treatment, which remained until now our preferred strategy of treatment. On the other hand, our current minimalist surgical method constitutes an evolutionary change of the awake LVRS that we have initially proposed in 2006 (14). Clinical data and radiologic findings of all potential candidates for LVRS are discussed within a multidisciplinary panel including thoracic surgeons, pulmonologists and radiologists in order to optimize the selection of surgical candidates and plan the optimal treatment strategy. In addition, all operated patients are encouraged to undergo a postoperative rehabilitation program preferentially performed within an in-hospital regimen in satellite specialized centers.

\section{Anesthesia}

The anesthesia protocol has gradually changed from preference of thoracic epidural anesthesia without sedation and under spontaneous ventilation (awake LVRS) (15), to adoption of intercostal block/paravertebral block with target control propofol (TCI) sedation and insertion of a supraglottic device (iGel-Intersurgical, Wokingham, UK) to have the possibility to assist ventilation whenever needed, keep the patient unconscious throughout the procedure, and as yet maintain spontaneous/assisted ventilation (Table 1).

With the current protocol, conversion to general anesthesia with intubation has never been required and all procedures could be safely completed even in patients with pleural adhesions and in several oxygen-dependent subjects with $\mathrm{PaO}_{2}<50 \mathrm{mmHg}$ and very poor pulmonary 
Table 1 Evolutionary changes in anesthesia protocol between intubated and nonintubated LVRS methods at our institution

\begin{tabular}{lccr}
\hline & IVATS & & NIVATS \\
\cline { 3 - 4 } Anesthesia characteristics & Yes & Non-resectional awake & Non-resectional minimalist \\
\hline Tracheal intubation & GA \pm TEA & TEA & No laryngeal mask \\
Anesthesia protocol & GA & No & IBA \\
Sedation & Mechanical & Spontaneous (target control) \\
Ventilation & Yes & No & Spontaneous \\
Diaphragm paralysis & No & Yes & No \\
Consciousness & Yes & No & No \\
Amnesia & No & Yes & Yes/no \\
Coughing reflex & YTS, & Yes/no \\
\hline
\end{tabular}

LVRS, lung volume reduction surgery; IVATS, intubated video-assisted thoracic surgery; NIVATS, non-intubated video-assisted thoracic surgery; GA, general anesthesia; TEA, thoracic epidural analgesia; IBA, intercostal block analgesia.

function as indicated by an $\mathrm{FEV}_{1}<20 \%$ of the predicted value. Nonetheless, a fiberoptic bronchoscope and doublelumen tubes are kept available in the operating room for bynecessity conversion to general anesthesia with single-lung ventilation.

\section{Surgical technique}

The patient is placed in lateral decubitus position as for thoracotomy. The operating table is usually not flexed to assure a more comfortable position and facilitate spontaneous ventilation of the dependent lung. The video monitor is placed at the head of the table. Surgical access entails placement of a single operative port though a $3-\mathrm{cm}$ skin incision performed along the anterior axillary line at the 4th or 5th intercostal space, which is employed both for a $30^{\circ}$-angled camera and surgical instrumentation. If present, pleural adhesions are coagulated and cut with a harmonic scalpel (Ultracision, Johnson \& Johnson Medical, Pomezia, Italy).

The surgical procedure has been progressively simplified to facilitate surgical maneuvering through a single surgical access, reduce lung manipulation, which may increase risks of postoperative air leaks, and maximize the degree of volume reduction achieved in the targeted lobe/s. Most commonly, the lung is grasped by a curved endoscopic ring forceps and a thoracoscopic noncutting stapler (Endopath 45 , Johnson \& Johnson, Pomezia, Italy) is fired in sequence along the targeted lobe to obtain a large plication of the lung tissue in a way to reduce the overall lung volume by about $30 \%$ (Figures 1,2) (Table 2). In typical upperlobe predominant emphysema cases, use of a single access offered a straight visualization of all upper lobes regions and facilitated division of pleural adhesions, which on average, are more frequently encountered at the dorsal and apical sides of the upper lobes.

\section{Conclusions}

Several uncontrolled and randomized studies published within the last 2 decades have shown that resectional LVRS can be highly effective in improving respiratory function, exercise capacity and quality of life for up to several years in selected patients with severe emphysema.

Nonetheless, in a study reporting on data from the Society of Thoracic Surgeons database (41), only 528 patients underwent LVRS in USA along an 8.5-year period. This finding highlights that despite the existence of a significant number of potential candidates, LVRS has declined in use in recent years probably due to the significant morbidity rate reported in a large randomized study (6).

We hypothesize that the main triggering factors of LVRS-related morbidity are constituted by adverse effects of general-anesthesia with tracheal intubation and mechanical ventilation as well as to the use of a resectional LVRS method entailing deep non-anatomical resection of emphysematous lung tissue (15).

In an attempt of reducing LVRS-related morbidity, in recent years, an active investigation has been devoted to 

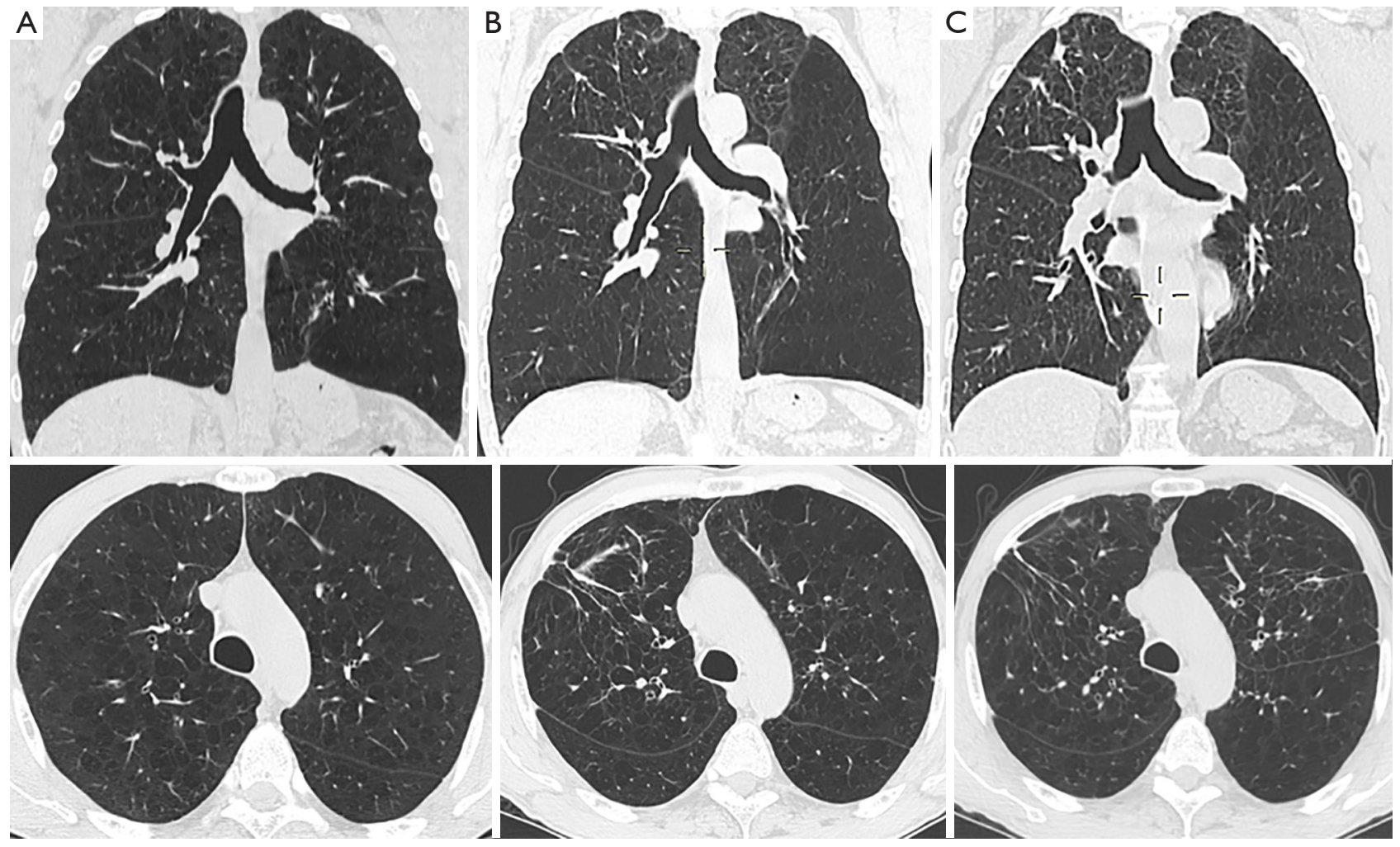

Figure 2 Typical HRCT findings of a patient undergoing staged unilateral minimalist LVRS. (A) Preoperative HRCT findings with upper lobe predominant severe non-bullous emphysema; (B) postoperative HRCT axial image after right minimalist LVRS; (C) postoperative radiological findings following left minimalist LVRS performed 15 months later. HRCT, high-resolution computed tomography; LVRS, lung volume reduction surgery.

Table 2 Evolutionary changes between standard, non-resectional and minimalist LVRS at our institution

\begin{tabular}{|c|c|c|c|}
\hline Characteristic & Resectional & Non-resectional awake & Non-resectional minimalist \\
\hline Number of thoracic ports & 4 & 3 & 1 \\
\hline Type of lung volume reduction & Non-anatomical lung resection & Non-anatomical lung plication & Non-anatomical lung plication \\
\hline Suture type & Staple, continuous & $\begin{array}{l}\text { Staple interrupted } \\
\text { (no-knife stapler) }\end{array}$ & $\begin{array}{l}\text { Staple interrupted } \\
\text { (no-knife stapler) }\end{array}$ \\
\hline Number of chest tubes & 2 & 2 & 1 \\
\hline
\end{tabular}

VATS, video-assisted thoracic surgery; LVRS, lung volume reduction surgery.

develop novel less and less invasive LVRS methods, which currently include surgical and bronchoscopic options carried out more often in a staged unilateral fashion, and possibly through nonintubated local-regional anesthesia protocols.
Our minimalist LVRS method represents the most recent evolutionary change of our previously described awake non-resectional method, and is aimed at summingup the advantages deriving from adoption of a uniportal surgical access combined with a novel non-resectional 
method and with an anesthesia protocol including sedation and use of simple intercostal blocks with maintenance of spontaneous ventilation. It holds promise but requires a thorough investigation of the preliminary results as well as a subsequent adequate comparison with the standard resectional technique.

In conclusion, we encourage a deeper investigation of the pros and cons of staged unilateral LVRS strategies as well as of redo-LVRS following bilateral treatment. Hopefully future studies taking into account the evolving spectrum of mini-invasive options, which include now surgical $(3,15)$ and nonsurgical $(16,17)$ non-resectional lung volume reduction treatment options, will probably allow to identify the most reliable methods allowing also to develop tailored treatment strategies according to the clinical functional status, the different radiologic characteristics of emphysema and of the personal preference of the patients.

In this way, we might be able to offer in the near future a comprehensive, multidisciplinary treatment of advanced emphysema aimed at assuring the maximal benefits with the lower risks according to a safe and reliable policy of personalized medicine.

\section{Acknowledgements}

Performed under appointment of the Minimalist Thoracic Surgery Research Group of the Tor Vergata University Policlinico with approval of the institutional ethical committee (No. 157/17).

\section{Footnote}

Conflicts of Interest: The authors have no conflicts of interest to declare.

\section{References}

1. Halbert RJ, Natoli JL, Gano A, et al. Global burden of COPD: systematic review and meta-analysis. Eur Respir J 2006;28:523-32.

2. O'Donnell DE, Revill SM, Webb KA. Dynamic hyperinflation and exercise intolerance in chronic obstructive pulmonary disease. Am J Respir Crit Care Med 2001;164:770-7.

3. Bingisser R, Zollinger A, Hauser M, et al. Bilateral volume reduction surgery for diffuse pulmonary emphysema by video-assisted thoracoscopy. J Thorac Cardiovasc Surg 1996;112:875-82.
4. Gelb AF, McKenna RJ Jr, Brenner M, et al. Lung function $5 \mathrm{yr}$ after lung volume reduction surgery for emphysema. Am J Respir Crit Care Med 2001;163:1562-6.

5. Ciccone AM, Meyers BF, Guthrie TJ, et al. Longterm outcome of bilateral lung volume reduction in 250 consecutive patients with emphysema. J Thorac Cardiovasc Surg 2003;125:513-25.

6. Fishman A, Martinez F, Naunheim K, et al. A randomized trial comparing lung-volume-reduction surgery with medical therapy for severe emphysema. N Engl J Med 2003;348:2059-73.

7. Pompeo E, Marino M, Nofroni I, et al. Reduction pneumoplasty versus respiratory rehabilitation in severe emphysema: a randomized study. Pulmonary Emphysema Research Group. Ann Thorac Surg 2000;70:948-53.

8. Keenan RJ, Landreneau RJ, Sciurba FC, et al. Unilateral thoracoscopic surgical approach for diffuse emphysema. J Thorac Cardiovasc Surg 1996;111:308-15.

9. Kotloff RM, Tino G, Palevsky HI, et al. Comparison of short-term functional outcomes following unilateral and bilateral lung volume reduction surgery. Chest 1998;113:890-5.

10. Oey IF, Waller DA, Bal S, et al. Lung volume reduction surgery--a comparison of the long term outcome of unilateral vs. bilateral approaches. Eur J Cardiothorac Surg 2002;;22:610-4.

11. Lowdermilk GA, Keenan RJ, Landreneau RJ, et al. Comparison of clinical results for unilateral and bilateral thoracoscopic lung volume reduction. Ann Thorac Surg. 2000;69:1670-4.

12. Serna DL, Brenner M, Osann KE, et al. Survival after unilateral versus bilateral lung volume reduction surgery for emphysema. J Thorac Cardiovasc Surg 1999;118:1101-9.

13. Pompeo E, Mineo TC. Long-term staged versus onestage bilateral thoracoscopic reduction pneumoplasty. Eur J Cardiothorac Surg 2002;21:627-33.

14. Mineo TC, Pompeo E, Mineo D, et al. Awake nonresectional lung volume reduction surgery. Ann Surg 2006;243:131-6.

15. Pompeo E, Rogliani P, Tacconi F, et al: Randomized comparison of awake nonresectional versus nonawake resectional lung volume reduction surgery. J Thorac Cardiovasc Surg 2012;143:47-54.

16. Sciurba FC, Ernst A, Herth FJ, et al. A randomized study of endobronchial valves for advanced emphysema. N Engl J Med. 2010; 363:1233-44.

17. Slebos DJ, Klooster K, Ernst A, et al. Bronchoscopic lung 
volume reduction coil treatment of patients with severe heterogeneous emphysema. Chest 2012;142:574-82.

18. Snell G, Herth FJF, Hopkins P, et al: Bronchoscopic thermal vapor ablation therapy in the management of heterogeneous emphysema. Eur Respir J 2012;39: 1326-33.

19. Choong CK, Macklem PT, Pierce JA, et al. Airway bypass improves the mechanical properties of explanted emphysematous lungs. Am J Respir Crit Care Med 2008;178:902-5.

20. Shah PL, Slebos DJ, Cordoso PFG, et al. Bronchoscopic lung-volume reduction with Exhale airways stents for emphysema (EASE trial): randomised, sham controlled, multicentre trial. Lancet 2011;378:997-1005.

21. Pompeo E, Rogliani P, Palombi L, et al. for the Awake Thoracic Surgery Research Group (ATSRG). The complex care of severe emphysema: role of awake lung volume reduction surgery. Ann Transl Med 2015;3:108.

22. McKenna RJ, Brenner M, Fishel RJ, et al. Patient selection criteria for lung volume reduction surgery. J Thorac Cardiovasc Surg 1997;114:957-64.

23. Mineo TC, Pompeo E, Mineo D, et al. Results of unilateral lung volume reduction surgery in patients with distinct heterogeneity of emphysema between-lungs. J Thorac Cardiovasc Surg 2005;129:73-9.

24. Pompeo E, Sergiacomi G, Nofroni I, et al. Morphologic grading of emphysema is useful in the selection of candidates for unilateral or bilateral reduction pneumoplasty. Eur J Cardiothorac Surg 2000;17:680-6.

25. Weder W, Tutic M, Lardinois D, et al. Persistent benefit from lung volume reduction surgery in patients with homogeneous emphysema. Ann Thorac Surg 2009;87:229-36.

26. National Emphysema Treatment Trial Research Group, Fishman A, Fessler H, et al. Patients at high risk of death after lung-volume reduction surgery. N Engl J Med 2001;345:1075-83.

27. Ingenito EP, Evans RB, Loring SH, et al. Relation between preoperative inspiratory lung resistance and outcome of lung volume reduction surgery for emphysema. N Engl J Med 1998;338:1181-5.

28. Washko GR, Criner GJ, Mohsenifar Z, et al. Computed tomographic-based quantification of emphysema and correlation to pulmonary function and mechanics. COPD 2008;5:177-86.

29. Criner GJ, Cordova F, Sternberg AL, et al. The National Emphysema Treatment Trial (NETT) Part II: Lessons Learned about Lung Volume Reduction Surgery. Am J Respir Crit Care Med 2011;184:881-93.
30. Hazelrigg SR, Boley TM, Magee MJ, et al. Comparison of staged thoracoscopy and median sternotomy for lung volume reduction. Ann Thorac Surg 1998;66:1134-9.

31. Brenner M, McKenna RJ Jr, Gelb AF, et al. Rate of FEV1 change following lung volume reduction surgery. Chest 1998;113:652-9.

32. Becker MD, Berkmen YM, Austin JH, et al. Lung volumes before and after lung volume reduction surgery. Am J Respir Crit Care Med 1998;157:1593-9.

33. Rathinam S, Oey I, Steiner M, et al. The role of the emphysema multidisciplinary team in a successful lung volume reduction surgery programme. Eur J Cardiothorac Surg 2014;46:1021-6.

34. Zhang M, Wang H, Pan XF, et al. Staged bilateral singleport thoracoscopic lung volume reduction surgery: A report of 11 cases. Exp Ther Med 2016;12:2851-4.

35. Crosa-Dorado VL, Pomi J, Perez-Penco EJ. Treatment of dyspnea in emphysema pulmonary remodeling: hemo and pneumostatic suturing of the emphysematous lung. Res Surg 1992;4:1-4.

36. Swanson SJ, Mentzer SJ, DeCamp MM, et al. No-cut thoracoscopic lung plication: a new technique for lung volume reduction surgery. J Am Coll Surg 1997;185:25-32.

37. Tacconi F, Pompeo E, Mineo TC. Duration of air leak is reduced after awake nonresectional lung volume reduction surgery. Eur J Cardiothorac Surg 2009;35:822-8.

38. Pompeo E, Mineo TC. Two-year improvement in multidimensional body mass index, airflow obstruction, dyspnea and exercise capacity index after nonresectional lung volume reduction surgery in awake patients. Ann Thorac Surg 2007;84:1862-9.

39. Tacconi F, Pompeo E, Forcella D, et al. Lung volume reduction reoperations. Ann Thorac Surg 2008;85:1171-7.

40. Kostron A, Horn-Tutic M, Franzen D, et al. Repeated lung volume reduction surgery is successful in selected patients. Eur J Cardiothorac Surg 2015;48:710-5.

41. Decker MR, Leverson GE, Jaoude WA, et al. Lung volume reduction surgery since the National Emphysema Treatment Trial: study of Society of Thoracic Surgeons Database. J Thorac Cardiovasc Surg 2014;148:2651-8.e1.

Cite this article as: Pompeo E, Rogliani P, Cristino B, Fabbi E, Dauri M, Sergiacomi G. Staged unilateral lung volume reduction surgery: from mini-invasive to minimalist treatment strategies. J Thorac Dis 2018;10(Suppl 23):S2754-S2762. doi: 10.21037/jtd.2018.05.171 